\title{
Simplified Brillouin fiber slow light systems in loss regime using step current modulation
}

\author{
Sanghoon Chin and Luc Thévenaz \\ Ecole Polytechnique Fédérale de Lausanne, STI-GR-SCI-LT, Station 11, CH-1015 Lausanne, Switzerland \\ sanghoon.chin@epfl.ch
}

\begin{abstract}
We propose a simple technique to realize Brillouin slow light in nearly transparent regime. A current-modulated semiconductor laser by a step function is used as Brillouin pump to generate a Brillouin loss doublet.
\end{abstract}

OCIS codes: (060.4370) Nonlinear optics, fibers; (290.5900) Scattering, stimulated Brillouin; (350.5500) Propagation

Recently, extensive investigations on Brillouin slow light in optical fibers have been theoretically and experimentally carried out in the photonics community, since stimulated Brillouin scattering (SBS) in optical fibers has shown an unmatched flexibility for tailoring the spectrum of complex optical resonances. The spectral broadening of SBS resonances by direct current modulation with Gaussian noise has been proposed to make Brillouin slow light system suitable for high capacity data streams [1]. However, it was soon identified that properly engineered dispersion properties of the actively modified medium response can produce signal delays with a reduced signal distortion [2], so that various direct current modulation schemes have been demonstrated to optimize the Brillouin slow light performance.

In this paper, we experimentally demonstrate a new direct-current modulation technique in order to generate a Brillouin gain/loss doublet in optical fibers. The injection current applied to a semiconductor laser was directly modulated by a square pulse waveform. The laser basically operates at the bias level when the pulse is in off-states, but the step increase of the injection current during on-states causes a shift in the laser frequency. In consequence, the laser frequency periodically alternates at the modulation frequency. However, as a result of the system linearity the effective Brillouin spectrum is given by the superposition of the individual spectra accumulated along the fiber. It means that the modulated laser is actually identical to a two-tone pump source as seen by the Brillouin slow light system. This way a Brillouin loss doublet is simply generated, as shown in Fig. 1, without using any external modulator. The spectral distance between the two peaks and the spectral width of each resonance were measured to be $322 \mathrm{MHz}$ and $88 \mathrm{MHz}$, respectively. In this type of Brillouin slow light systems the signal bandwidth is usually determined by the difference between these two widths [3]. However, this system can easily adapt to any broadband signals, by simply increasing the modulation depth, so further separating the two Brillouin resonances. Linewidth broadening of the laser was also clearly observed since transient phase fluctuations occur in the laser active region due to the carrier-induced and thermal refractive index change. However, it allows this system to be operated in the optimal delay-bandwidth product conditions. Fig. 2 shows the normalized time waveforms of the delayed pulses for different pump powers. A signal pulse with duration of 6 ns was continuously delayed up to $2.04 \mathrm{~ns}$, corresponding to a fractional delay of 0.34 for a pump power of $260 \mathrm{~mW}$.

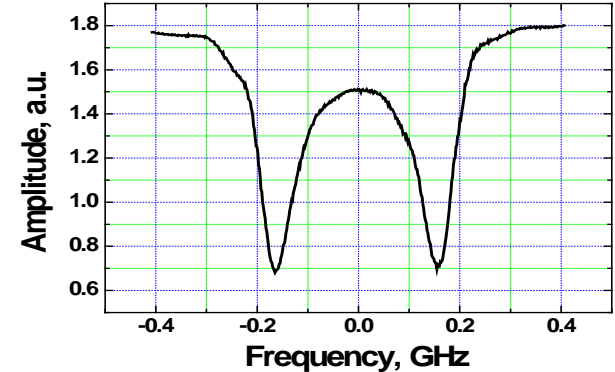

Fig. 1. Amplitude variation of the probe laser after propagating through the fiber, clearly showing a Brillouin doublet.

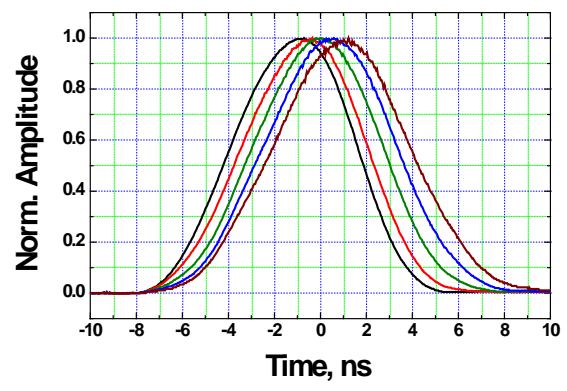

Fig. 2. Time waveforms of delayed signal pulses for different pump powers after propagating through the fiber.

\section{References}

[1] M. Gonzalez-Herráez, K. Y. Song, and L. Thévenaz, "Arbitrary-bandwidth Brillouin slow light in optical fibers," Opt. Express 14, 13951400 (2006).

[2] A. Zadok, A. Eyal, and M. Tur, "Extended delay of broadband signals in stimulated Brillouin scattering slow light using synthesized pump chirp," Opt. Express 14, 8498-8505 (2006).

[3] Z. Zhu and D. J. Gauthier, "Nearly transparent SBS slow light in an optical fiber," Opt. Express 14, 7238-7245 (2006). 